http://dx.doi.org/10.32911/as.2017.v10.n2.164

Aporte Santiaguino. 10 (2), 2017: 211-224

ISSN 2070-836X

\title{
La inversión pública nacional, regional y local a nivel departamental y su incidencia en la reducción de la pobreza en el Perú, 2008 - 2015
}

National, Regional and Local Public Investment at departmental level and its impact on poverty reduction in Peru, 2008 - 2015

Jorge Manrique Cáceres ${ }^{1}$ y Jéssica Polonio Toledo ${ }^{1}$

\section{RESUMEN}

El objetivo del estudio fue investigar la incidencia de la inversión pública en sus tres niveles de gobierno (la inversión que realiza directamente el Gobierno Nacional, la inversión de los gobiernos regionales y la inversión de los gobiernos locales) sobre la reducción de la pobreza estructural para el caso peruano a partir de información departamental (2008-2015). Los resultados muestran que la incidencia de cada uno de estos niveles de gobierno sobre la pobreza estructural ha sido diferente, así encontramos que la inversión de los gobiernos locales ha sido la que más ha contribuido a la reducción de la pobreza, seguido por los programas nacionales; sin embargo, la inversión de los gobiernos regionales todavía no logra el resultado esperado y debe optimizarse. Se enfatiza la necesidad de implementar planes de desarrollo coordinado para mejorar la eficacia de la inversión pública. Al mismo tiempo, este trabajo en el aspecto académico, ha permitido emplear el Modelo de Datos de Panel como técnica econométrica de análisis y como mecanismo para suplir escasez de información.

Palabras clave: pobreza; inversión pública; Gobierno Nacional; gobiernos locales.

\begin{abstract}
The objective of the study was to investigate the impact of different levels of public investment (investment implement directly by National Government, Regional governments investment and local governments investment) on the reduction of structural poverty using subnational-level data for Peru (2008-2015). The results show that the incidence of each of these levels of government on structural poverty has been different,
\end{abstract}

1 Universidad Nacional Santiago Antúnez de Mayolo. Huaraz, Perú. 
so we find that local government investment has contributed most to poverty reduction, followed by the national programs; however, regional governments investment not yet achieved the desired result and must be optimized. The need to implement coordinated development plans to improve the effectiveness of public investment are emphasized. At the same time, this work in the academic aspect has allowed us to use Panel Data Model as an econometric analysis technique and as a mechanism to supply shortages of information.

Keywords: poverty; public investment; national government; regional government; local governments.

\section{INTRODUCCIÓN}

En el periodo 2008-2015, la inversión pública se incrementó de manera significativa, lo cual se explica básicamente por: i) la ejecución de un importante portafolio de mega proyectos de infraestructura; y, ii) el impulso de la inversión a nivel de los gobiernos subnacionales. ${ }^{2}$ Durante dicho periodo, la inversión pública en los tres niveles de gobierno (nacional, regional y local) mostró un importante crecimiento, lo que evidencia una orientación descentralizada del sistema, como se puede apreciar en la figura 1.

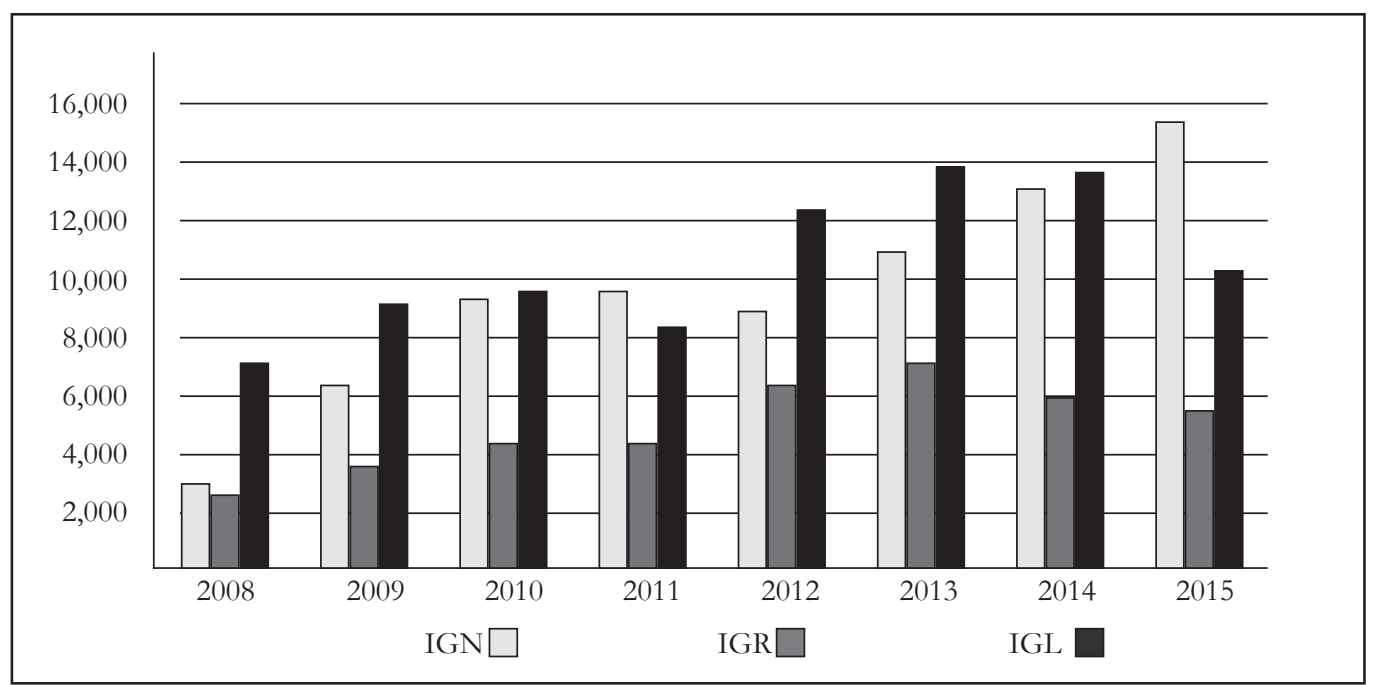

Figura 1. Evolución de la inversión pública por niveles de gobierno (millones de Soles) Fuente: Banco Central de Reserva del Perú - Información Regional

\footnotetext{
2 Marco Macroeconómico Multianual 2016-2018.
} 
En cuanto a la participación porcentual de la inversión del gobierno nacional, regional y local en la inversión pública total, la tabla 1 nos muestra que, a excepción de los años 2011 y 2015, esta se encuentra concentrada en el gobierno local, seguido por el Gobierno Nacional y gobierno regional. En promedio, durante el periodo de referencia, la inversión pública a cargo del gobierno local representó el 42,5 \% de la inversión pública total del país; la inversión nacional representó en promedio el 37 \% y, la inversión regional, el 20,5\% del total.

Tabla 1: Inversión pública por niveles de gobierno (Participación porcentual)

\begin{tabular}{ccccccccc}
\hline $\begin{array}{c}\text { Nivel de } \\
\text { Gobierno }\end{array}$ & 2008 & 2009 & 2010 & 2011 & 2012 & 2013 & 2014 & 2015 \\
\hline Nacional & 25.5 & 33.6 & 38.8 & 43.3 & 32.8 & 34.2 & 39.5 & 48.4 \\
Regional & 20.9 & 20.0 & 20.3 & 20.1 & 23.6 & 22.1 & 18.9 & 18.3 \\
Local & 53.6 & 46.4 & 40.9 & 36.6 & 43.6 & 43.7 & 41.6 & 33.3 \\
\hline Total & 100.0 & 100.0 & 100.0 & 100.0 & 100.0 & 100.0 & 100.0 & 100.0 \\
\hline
\end{tabular}

Fuente: Banco Central de Reserva del Perú - Información Regional

Resulta interesante, evaluar la evolución de la inversión pública (en los tres niveles de gobierno) con la evolución de la pobreza, en un esfuerzo por analizar la posible incidencia de esta forma de intervención pública. En este sentido, cabe destacar que: «En la medida en que la inversión pública sea capaz de afectar la productividad de la mano de obra y capital privados (modificando la dotación de capital físico y humano en una región) será capaz de afectar la capacidad de generación de ingresos de las familias y, con esto, su nivel de pobreza» (UP, 2010: 7).

Como se observa en la figura 2 , la tasa de pobreza monetaria ${ }^{3}$ en nuestro país se ha reducido notoriamente en los últimos años. En el año 2015, el 21,8 \% de la población del país, que equivale en cifras absolutas a 6 millones 782 mil personas, se encontraban en situación de pobreza. Al comparar con el nivel obtenido en el año 2008 (en el que el 37,3 \% de la población era pobre), la incidencia de la pobreza disminuyó en 15,5 \% durante este periodo.

En cuanto a la pobreza por Necesidades Básicas Insatisfechas (NBI) ${ }^{4}$, en el año 2015,

\footnotetext{
3 Se considera como pobres monetarios a las personas que residen en hogares cuyo gasto per cápita es insuficiente para adquirir una canasta básica de alimentos y no alimentos (vivienda, vestido, educación, salud, transporte, etc.). (Feres y Mancero, 2001).

4 Se considera población con al menos una Necesidad Básica Insatisfecha, aquella que padece de al menos una de las cinco siguientes carencias: i) a los que residen en viviendas con características físicas inadecuadas; ii) a la población que pertenece a hogares en viviendas en hacinamiento; iii) a los que residen en viviendas sin ningún tipo de servicio higiénico; iv) a la población en hogares con niños y niñas de 6 a 12 años de edad que no asisten al colegio; v) población en hogares con alta dependencia económica. Ídem.
} 
el 19,4 \% de la población del país habitaba en hogares con al menos una NBI, respecto a lo registrado el año 2008 (28,9 \%) disminuyó en 9,5 puntos porcentuales.

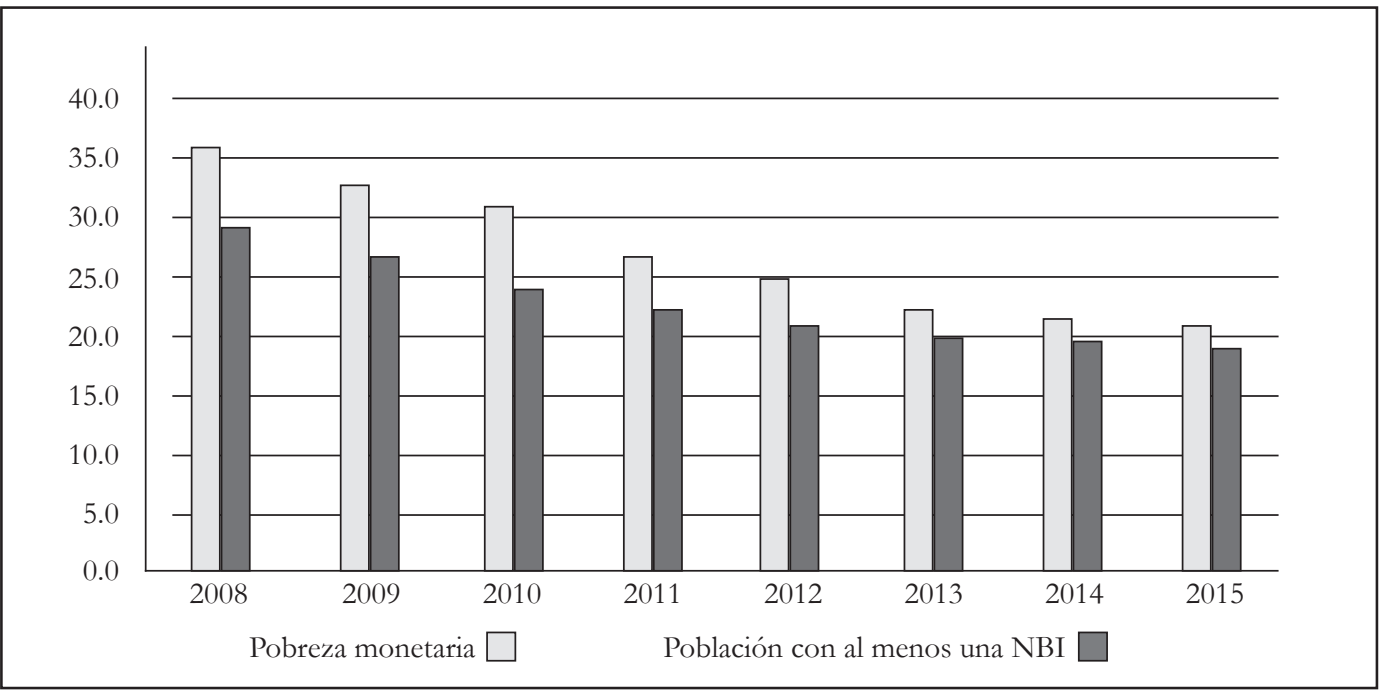

Figura 2. Tasa de pobreza, 2008 - 2015 (\% Población total)

Fuente: Instituto Nacional de Estadística e Informática - Estadísticas Sociales

Entre los antecedentes del presente trabajo podemos citar al desarrollado por Ford y Paredes (2015), quienes sostienen que la inversión pública rural tiene un efecto importante sobre la reducción de la pobreza. Por un lado, la inversión pública del Gobierno Nacional realizada en fortalecimiento del capital humano, inversiones en conectividad, acceso a mercados, riego, caminos, telecomunicaciones, entre otros, tienen un efecto significativo sobre la reducción de la pobreza rural. Por otro lado, en el rol de los gobiernos subnacionales, se encuentra que la descentralización de los recursos de la inversión pública rural tiene un efecto significativo sobre la reducción de la pobreza, pero que este depende en gran medida del sector analizado.

También recurrimos al trabajo desarrollado por el Instituto Peruano de Economía (2006), cuyo estudio revela que la inversión en infraestructura de servicios públicos contribuye a la reducción de la pobreza. La inversión pública en infraestructura afecta directamente a los consumidores a través de un mayor bienestar y permite a las empresas elevar su nivel de producción y contribuir de esta manera al crecimiento económico; reduciendo así la pobreza. Se evidencia que son las regiones que incrementan su provisión de infraestructura las que encuentran un menor nivel de pobreza. 
La realización del presente trabajo tiene su justificación en dos aspectos principales: a) conocer con mayor precisión la efectividad de este flujo de inversión pública, como también el alcance de sus resultados, más aun teniendo en cuenta el gran interés que han despertado las teorías que respaldan a la inversión pública como un factor fundamental para el crecimiento económico y la reducción de la pobreza; y b) realizar un análisis más dinámico respecto a este tema utilizando un modelo de datos de panel, lo cual además de enriquecer el estudio, permitió captar mejor la relación entre la inversión pública (nacional, regional y local) a nivel departamental y la reducción de la pobreza.

El objetivo general de este estudio es determinar la incidencia de la inversión pública nacional, regional y local a nivel departamental en la reducción de la pobreza en el Perú, en el periodo 2008-2015.

Dentro de todo este escenario nacional podemos intentar una respuesta anticipada en los siguientes términos: La inversión pública nacional, regional y local a nivel departamental incide positivamente en la reducción de la pobreza en el Perú, en el periodo 2008-2015.

\section{La inversión pública, el crecimiento económico y la reducción de la pobreza}

La inversión pública es capaz de afectar directamente la dotación de capital físico y humano de cada región y, a través de ello, la productividad de la mano de obra y capital privados, contribuyendo así al crecimiento económico (UP, 2010). Este crecimiento, se traduce generalmente en un aumento de los ingresos del hogar y las reducciones en la incidencia de la pobreza 5 . Teniendo como referente sucesivos reportes sobre el desarrollo mundial publicado anualmente por el Banco Mundial, esta asociación se va reforzando en evidencia año a año (Adrianzén, 2013). Por tanto, es posible afirmar que la inversión pública ha desempeñado un rol activo en la consecución de resultados socioeconómicos positivos.

\section{La inversión pública en infraestructura como medio para reducir la pobreza}

Los mecanismos de transmisión de los efectos de la inversión pública sobre la economía son importantes (Agénor, 2006). En primer lugar, los beneficiados son los hogares, quienes pueden acceder a una fuente de trabajo que les permite percibir un ingreso y obtener mayor bienestar producto de los servicios relacionados con una nueva in-

5 En general, existe un consenso en la literatura reciente sobre la relación entre el crecimiento económico y la reducción de la pobreza en los países en desarrollo. Aunque los ingresos de fuentes de datos de crecimiento no son iguales y diferentes medidas de pobreza pueden producir resultados diversos, estos resultan lo suficientemente consistentes que nos permiten sugerir que el crecimiento económico disminuye la incidencia de la pobreza. 
fraestructura y, en segundo lugar, la mayor provisión de infraestructura también afecta a las empresas a través de dos mecanismos, la reducción de costos y la posibilidad de ampliar los mercados tanto internos como externos (IPE, 2006). Además de una reducción de la pobreza debido al efecto indirecto que tiene la inversión pública en infraestructura por la vía de un mayor crecimiento económico, existen vías directas por las que la inversión en infraestructura puede contribuir a disminuir la incidencia de la pobreza (Durán y Saavedra, 2014).

\section{Inversión Pública Subnacional y reducción de la pobreza}

Oates (1972) sostiene que el efecto positivo de la descentralización fiscal sobre el bienestar social se origina en la cercanía de la toma de decisiones en materia de gasto público a nivel local que genera el proceso, lo cual permite un mejor alineamiento de preferencias (presumiblemente heterogéneas) y genera ganancias definitivas de eficiencia social.

\section{MATERIALES Y MÉTODOS}

En lo referente al tipo de investigación, esta es aplicada, descriptiva y correlacional. El diseño es no experimental y el análisis se realizó de manera transversal y longitudinal simultáneamente. La población en estudio son las variables: pobreza e inversión pública nacional, regional y local a nivel de los 24 departamentos del Perú (considerando a Lima y el Callao como uno solo), en el periodo 2008 - 2015. Los datos usados son de frecuencia anual, a nivel departamental.

La información referente a la inversión pública se obtuvo del Banco Central de Reserva del Perú (BCRP), mientras que la información relativa a pobreza se recopiló del Instituto Nacional de Estadística e Informática (INEI). Se recopiló información estadística de las estadísticas anuales a nivel departamental. Para el análisis y tratamiento de la información, se utilizó un modelo de Datos de Panel, el cual permitió realizar el análisis de corte transversal y longitudinal de manera simultánea. Asimismo, se usó el programa Eviews 8.1, para el procesamiento de los datos.

\section{RESULTADOS}

El objetivo del análisis empírico es determinar la incidencia de la inversión pública (a nivel nacional, regional y local) en la reducción de la pobreza del Perú. Dicho análisis se realiza a nivel departamental, durante el periodo 2008-2015. 
En primer lugar, se analiza el efecto conjunto de la inversión pública nacional, regional y local a nivel departamental en la reducción de la pobreza y, seguidamente, se analiza cada uno de los casos de manera desagregada. Para las distintas estimaciones se han tomado las series anuales por departamentos de la Inversión del Gobierno Nacional (IGN), la Inversión de los Gobiernos Regionales (IGR), la Inversión de los Gobiernos Locales (IGL) y la Pobreza según NBI (Pob).

Análisis conjunto:

La heterogeneidad encontrada en las series estadísticas, garantiza una de las condiciones del uso de Datos de Panel. Asimismo, mediante la prueba Durbin-Wu-Hausman se establece que el modelo de Datos de Panel más adecuado en este caso es el Modelo de Panel Estático, por tener variables estrictamente exógenas. En base a ello, se evalúa si debe aplicarse un modelo de efectos fijos o un modelo de efectos aleatorios.

Tabla 2. Modelos de efectos

\begin{tabular}{|c|c|c|c|c|c|c|c|c|c|}
\hline \multicolumn{5}{|c|}{ Modelos de Efectos Fijos } & \multicolumn{5}{|c|}{ Modelo de Efectos Aleatorios } \\
\hline \multicolumn{5}{|c|}{$\begin{array}{l}\text { Dependent Variable: LNPOB } \\
\text { Method: Panel Least Squares } \\
\text { Date: } 03 / 05 / 17 \text { Time: } 07: 12 \\
\text { Sample: } 20082015 \\
\text { Periods included: } 8 \\
\text { Cross-sections included: } 24 \\
\text { Total panel (balanced) observations: } 192\end{array}$} & \multicolumn{5}{|c|}{$\begin{array}{l}\text { Dependent Variable: LNPOB } \\
\text { Method: Panel EGLS (Cross-section random effects) } \\
\text { Date: } 03 / 05 / 17 \text { Time: } 07: 23 \\
\text { Sample: } 20082015 \\
\text { Periods included: } 8 \\
\text { Cross-sections included: } 24 \\
\text { Total panel (balanced) observations: } 192 \\
\text { Swamy and Arora estimator of component variances } \\
\end{array}$} \\
\hline Variable & Coefficient & Std. Error & t-Statistic & Prob. & Variable & Coefficient & Std. Error & t-Statistic & Prob. \\
\hline $\begin{array}{l}\text { LNIGN } \\
\text { LNIGR } \\
\text { LNIGL } \\
\quad \text { C }\end{array}$ & $\begin{array}{r}-0.030592 \\
0.017518 \\
-0.277371 \\
8.846713\end{array}$ & $\begin{array}{l}0.021611 \\
0.023507 \\
0.035949 \\
0.642770\end{array}$ & $\begin{array}{r}-1.415528 \\
0.745206 \\
-7.715712 \\
13.76343\end{array}$ & $\begin{array}{l}0.1588 \\
0.4572 \\
0.0000 \\
0.0000\end{array}$ & $\begin{array}{l}\text { LNIGN } \\
\text { LNIGR } \\
\text { LNIGL } \\
\quad \mathrm{C}\end{array}$ & $\begin{array}{r}-0.032918 \\
0.010094 \\
-0.240879 \\
8.250492\end{array}$ & $\begin{array}{l}0.021121 \\
0.023366 \\
0.034360 \\
0.626300\end{array}$ & $\begin{array}{r}-1.558575 \\
0.432004 \\
-7.010354 \\
13.17338\end{array}$ & $\begin{array}{l}0.1208 \\
0.6662 \\
0.0000 \\
0.0000\end{array}$ \\
\hline \multicolumn{5}{|c|}{ Effects Specification } & \multicolumn{5}{|c|}{ Effects Specification } \\
\hline \multicolumn{5}{|c|}{ Cross-section fixed (dummy variables) } & \multicolumn{3}{|l|}{$\begin{array}{l}\text { Cross-section random } \\
\text { Idiosyncratic random }\end{array}$} & $\begin{array}{l}0.478579 \\
0.151632\end{array}$ & $\begin{array}{l}0.9088 \\
0.0912\end{array}$ \\
\hline $\begin{array}{l}\text { Adjusted R-squared } \\
\text { S.E. of regression }\end{array}$ & $\begin{array}{l}0.905858 \\
0.151632\end{array}$ & \multirow{2}{*}{\multicolumn{2}{|c|}{$\begin{array}{l}\text { Mean dependent var } \\
\text { S.D. dependent var } \\
\text { Akaike info criterion } \\
\text { Schwarz criterion } \\
\text { Hannan-Quinn criter. } \\
\text { Durbin-Watson stat }\end{array}$}} & \multirow{2}{*}{$\begin{array}{r}3.271054 \\
0.494194 \\
-0.805026 \\
-0.346941 \\
-0.619498 \\
0.918742\end{array}$} & & Weighted & Statistics & & \\
\hline $\begin{array}{l}\text { Sum squared resid } \\
\text { Log likelihood } \\
\text { F-statistic } \\
\text { Prob(F-statistic) }\end{array}$ & $\begin{array}{l}3.793701 \\
104.2825 \\
71.68627 \\
0.000000\end{array}$ & & & & $\begin{array}{l}\text { R-squared } \\
\text { Adjusted R-squared } \\
\text { S.E. of regression }\end{array}$ & $\begin{array}{l}0.250706 \\
0.238749 \\
0.155410\end{array}$ & $\begin{array}{l}\text { Mean depend } \\
\text { S.D. depende } \\
\text { Sum squared }\end{array}$ & $\begin{array}{l}\text { ent var } \\
\text { nt var } \\
\text { resid }\end{array}$ & $\begin{array}{l}0.364142 \\
0.178121 \\
4.540652\end{array}$ \\
\hline \multirow{3}{*}{ Prob(F-statistic) } & & & & & $\begin{array}{l}\text { F-statistic } \\
\text { Prob(F-statistic) }\end{array}$ & $\begin{array}{l}20.96761 \\
0.000000\end{array}$ & Durbin-Watso & n stat & 0.729259 \\
\hline & & & & & \multicolumn{5}{|c|}{ Unweighted Statistics } \\
\hline & & & & & $\begin{array}{l}\text { R-squared } \\
\text { Sum squared resid }\end{array}$ & $\begin{array}{r}-0.332719 \\
62.16790\end{array}$ & $\begin{array}{l}\text { Mean depend } \\
\text { Durbin-Watso }\end{array}$ & $\begin{array}{l}\text { ent var } \\
\text { in stat }\end{array}$ & $\begin{array}{l}3.271054 \\
0.053264\end{array}$ \\
\hline
\end{tabular}


Test de Hausman

\begin{tabular}{|c|c|c|c|c|}
\hline Test Summary & & -Sq. Statistic & Chi-Sq. d.f. & Prob. \\
\hline Cross-section random & & 12.487260 & 3 & 0.0059 \\
\hline \multicolumn{5}{|c|}{ Cross-section random effects test comparisons: } \\
\hline Variable & Fixed & Random & $\operatorname{Var}$ (Diff.) & Prob. \\
\hline LNIGN & -0.030592 & -0.032918 & 0.000021 & 0.6115 \\
\hline LNIGR & 0.017518 & 0.010094 & 0.000007 & 0.0040 \\
\hline LNIGL & -0.277371 & -0.240879 & 0.000112 & 0.0006 \\
\hline
\end{tabular}

De acuerdo a los resultados del Test de Hausman, el modelo aceptado es el modelo de Efectos Fijos. Prob $=0,0059<0,05$

El coeficiente que acompaña a la IGN (Inversión del Gobierno Nacional en cada departamento), tiene un valor negativo lo cual confirma la hipótesis de que a un aumento porcentual de la IGN en $1 \%$, permite una reducción de la pobreza (medida por el método de la NBI) en un 0.0306 \%. Acápite aparte, merece especial atención la Inversión realizada por los Gobierno Regionales (IGR), que cuando interactúa en forma conjunta con los diferentes niveles de inversión (IGN, IGR y IGL), los resultados obtenidos no están contribuyendo a la reducción de la pobreza. La Inversión de los gobiernos locales, por su parte, incide en la reducción de la pobreza, el valor del estimado de -0.02773 nos indica que por cada $1 \%$ de aumento de la inversión, la reducción de la pobreza es del $0,02773 \%$.

Análisis Desagregado:

Inversión del Gobierno Nacional 
Tabla 3. Modelos de efectos

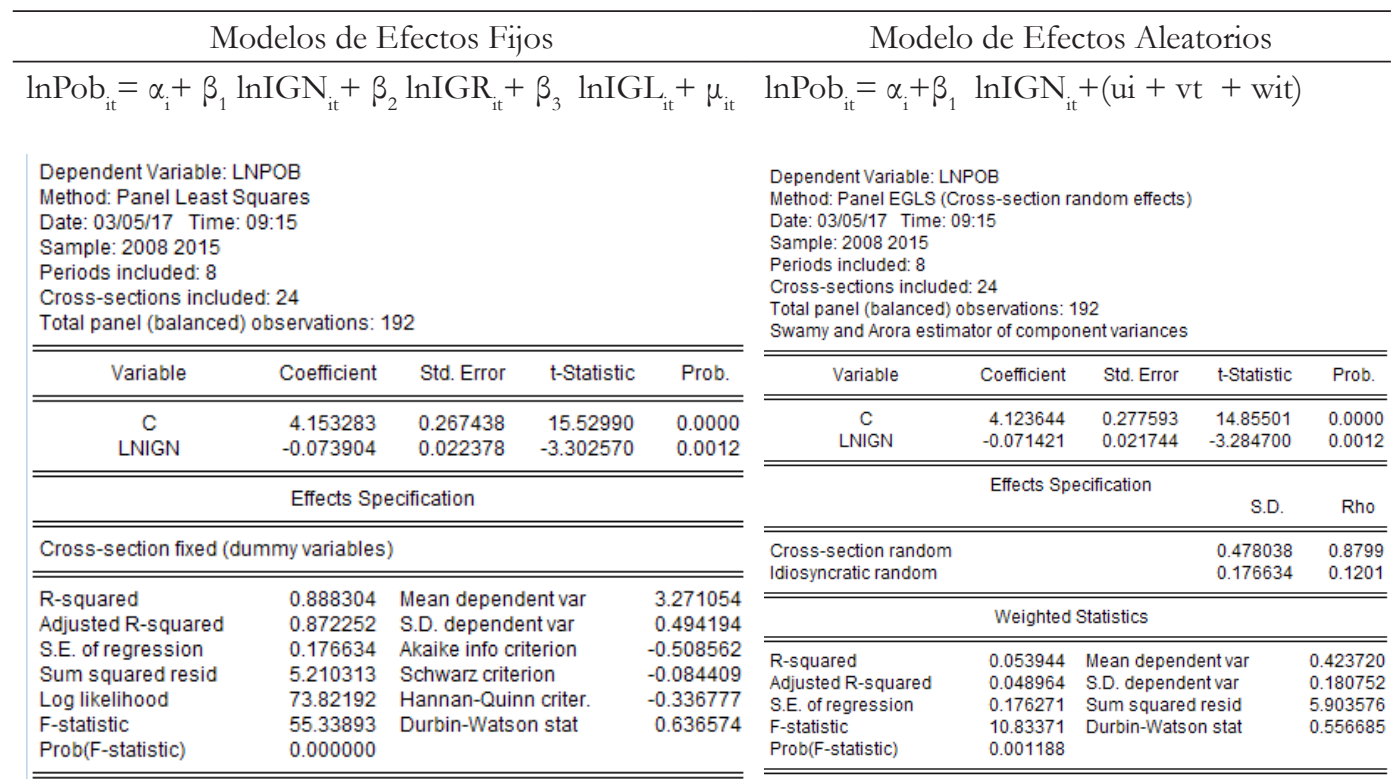

$\ln \mathrm{Pob}_{\mathrm{it}}=4.1532-0.0739 \ln \mathrm{IGN}_{\mathrm{it}}$ $\ln \mathrm{Pob}_{\mathrm{it}}=4.1236-0.0714 \ln \mathrm{IGN}_{\mathrm{it}}$

Test de Hausman

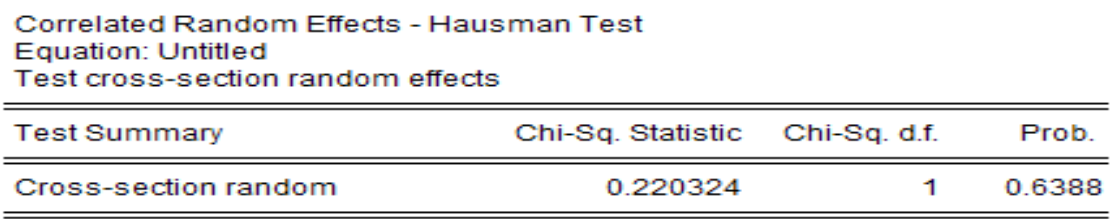

Cross-section random effects test comparisons:

\begin{tabular}{ccccc} 
Variable & Fixed & Random & Var(Diff.) & Prob. \\
\hline \hline LNIGN & -0.073904 & -0.071421 & 0.000028 & 0.6388 \\
\hline \hline
\end{tabular}

De acuerdo a los resultados del Test de Hausman, el modelo aceptado es el modelo de Efectos Aleatorios. Prob $=0,6388>0,05$

La Inversión del Gobierno Nacional en relación con la reducción de la pobreza, muestra resultados acordes a lo estipulado por la teoría. El estimador asociado nos indica que por cada $1 \%$ de aumento en la IGN su contribución a la reducción de la pobreza es del $0.0714 \%$.

Inversión de los gobiernos regionales 
Tabla 4. Modelos de efectos

\begin{tabular}{|c|c|c|c|c|c|c|c|c|c|}
\hline \multicolumn{5}{|c|}{ Modelos de Efectos Fijos } & \multicolumn{5}{|c|}{ Modelo de Efectos Aleatorios } \\
\hline \multicolumn{5}{|c|}{$\ln \mathrm{Pob}_{\mathrm{it}}=\alpha \mathrm{i}+\beta_{1} \ln \mathrm{IGR}_{\mathrm{it}}+\mu_{\mathrm{it}}$} & \multicolumn{5}{|c|}{$\ln \mathrm{Pob}_{\mathrm{it}}=\alpha_{\mathrm{i}}+\beta_{1} \ln \mathrm{IGR}_{\mathrm{it}}+(\mathrm{ui}+\mathrm{vt}+\mathrm{wit})$} \\
\hline \multicolumn{5}{|c|}{$\begin{array}{l}\text { Dependent Variable: LNPOB } \\
\text { Method: Panel Least Squares } \\
\text { Date: } 03 / 05 / 17 \text { Time: } 09: 19 \\
\text { Sample: } 2008 \text { 2015 } \\
\text { Periods included: } 8 \\
\text { Cross-sections included: } 24 \\
\text { Total panel (balanced) observations: } 192\end{array}$} & \multicolumn{5}{|c|}{$\begin{array}{l}\text { Dependent Variable: } \text { LNPOB } \\
\text { Method: Panel ELLS (Cross-section random effects) } \\
\text { Date: } 0 \text { 3/05/17 Time: } 09: 20 \\
\text { Sample: } 20082015 \\
\text { Periods included: } 8 \\
\text { Cross-sections included: } 24 \\
\text { Total panel (balanced) observations: } 192 \\
\text { Swamy and Arora estimator of component variances }\end{array}$} \\
\hline Variable & Coefficient & Std. Error & t-Statistic & Prob. & Variable & Coefficient & Std. Error & t-Statistic & Prob. \\
\hline$\underset{\text { LNIGR }}{\mathrm{C}}$ & $\begin{array}{r}3.995033 \\
-0.060236\end{array}$ & $\begin{array}{l}0.290004 \\
0.024105\end{array}$ & $\begin{array}{r}13.77579 \\
-2.498927\end{array}$ & $\begin{array}{l}0.0000 \\
0.0134\end{array}$ & $\begin{array}{c}C \\
\text { LNIGR }\end{array}$ & $\begin{array}{r}4.006944 \\
-0.061227\end{array}$ & $\begin{array}{l}0.304462 \\
0.023994\end{array}$ & $\begin{array}{r}13.16073 \\
-2.551805\end{array}$ & $\begin{array}{l}0.0000 \\
0.0115\end{array}$ \\
\hline \multicolumn{5}{|c|}{ Effects Specification } & \multicolumn{5}{|c|}{ Effects Specification } \\
\hline \multirow{3}{*}{$\begin{array}{l}\text { R-squared } \\
\text { Adjusted R-squared } \\
\text { S.E. of regression } \\
\text { Sum squared resid } \\
\text { Log likelihood } \\
\text { F-statistic } \\
\text { Prob(F-statistic) }\end{array}$} & \multirow{3}{*}{$\begin{array}{l}0.885298 \\
0.868814 \\
0.178995 \\
5.350533 \\
71.27253 \\
53.70633 \\
0.000000 \\
\end{array}$} & \multirow{3}{*}{\multicolumn{2}{|c|}{$\begin{array}{l}\text { Mean dependent var } \\
\text { S.D. dependent var } \\
\text { Akaike info criterion } \\
\text { Schwarz criterion } \\
\text { Hannan-Quinn criter. } \\
\text { Durbin-Watson stat }\end{array}$}} & \multirow{3}{*}{$\begin{array}{r}3.271054 \\
0.494194 \\
-0.482006 \\
-0.057852 \\
-0.310220 \\
0.548456\end{array}$} & \multicolumn{2}{|l|}{$\begin{array}{l}\text { Cross-section random } \\
\text { Idiosyncratic random }\end{array}$} & \multicolumn{2}{|r|}{$\begin{array}{l}0.474143 \\
0.178995\end{array}$} & $\begin{array}{l}0.8753 \\
0.1247 \\
\end{array}$ \\
\hline & & & & & \multicolumn{5}{|c|}{ Weighted Statistics } \\
\hline & & & & & \multirow{2}{*}{$\begin{array}{l}\text { R-squared } \\
\text { Adjusted R-squared } \\
\text { S.E. of regression } \\
\text { F-statistic } \\
\text { Prob(F-statistic) } \\
\end{array}$} & \multirow{2}{*}{$\begin{array}{l}0.033275 \\
0.028187 \\
0.178610 \\
6.539804 \\
0.011329\end{array}$} & \multirow{2}{*}{\multicolumn{2}{|c|}{$\begin{array}{l}\text { Mean dependent var } \\
\text { S.D. dependent var } \\
\text { Sum squared resid } \\
\text { Durbin-Watson stat }\end{array}$}} & \multirow[t]{2}{*}{$\begin{array}{l}0.432753 \\
0.181182 \\
6.061279 \\
0.484958\end{array}$} \\
\hline & & & & & & & & & \\
\hline \multicolumn{5}{|c|}{$\ln \mathrm{Pob}_{\mathrm{it}}=3.9950-0.0602 \ln \mathrm{IGR}_{\mathrm{it}}$} & \multicolumn{5}{|c|}{$\ln \mathrm{Pob}_{\mathrm{it}}=4.0069-0.0612 \ln \mathrm{IGR}_{\mathrm{it}}$} \\
\hline
\end{tabular}

Test de Hausman

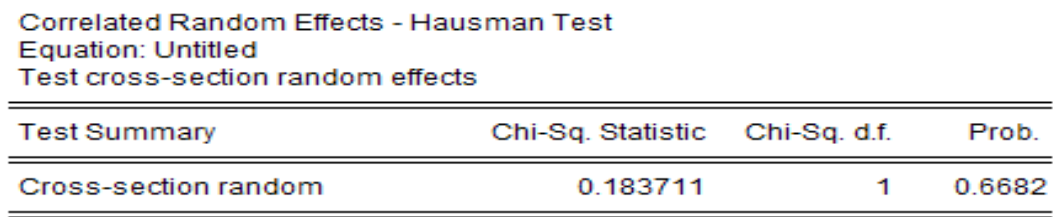

Cross-section random effects test comparisons:

\begin{tabular}{ccccc} 
Variable & Fixed & Random & Var(Diff.) & Prob. \\
\hline \hline LNIGR & -0.060236 & -0.061227 & 0.000005 & 0.6682 \\
\hline
\end{tabular}

De acuerdo a los resultados del Test de Hausman, el modelo aceptado es el modelo de Efectos Aleatorios. Prob $=0,6682>0,05$

El estimador asociado a la Inversión de los Gobiernos Regionales en relación con la reducción de la pobreza, nos indica que por cada $1 \%$ de aumento en la IGR, la contribución a la reducción de la pobreza es de 0,0612\%.

Inversión de los Gobiernos Locales

La tabla 5 muestra que el estimador asociado a la Inversión de los Gobiernos Locales en relación con la reducción de la pobreza, nos indica que por cada $1 \%$ de aumento en la IGL, la contribución a la reducción de la pobreza es de 0,2832\%. 
Tabla 5. Modelos de Efectos

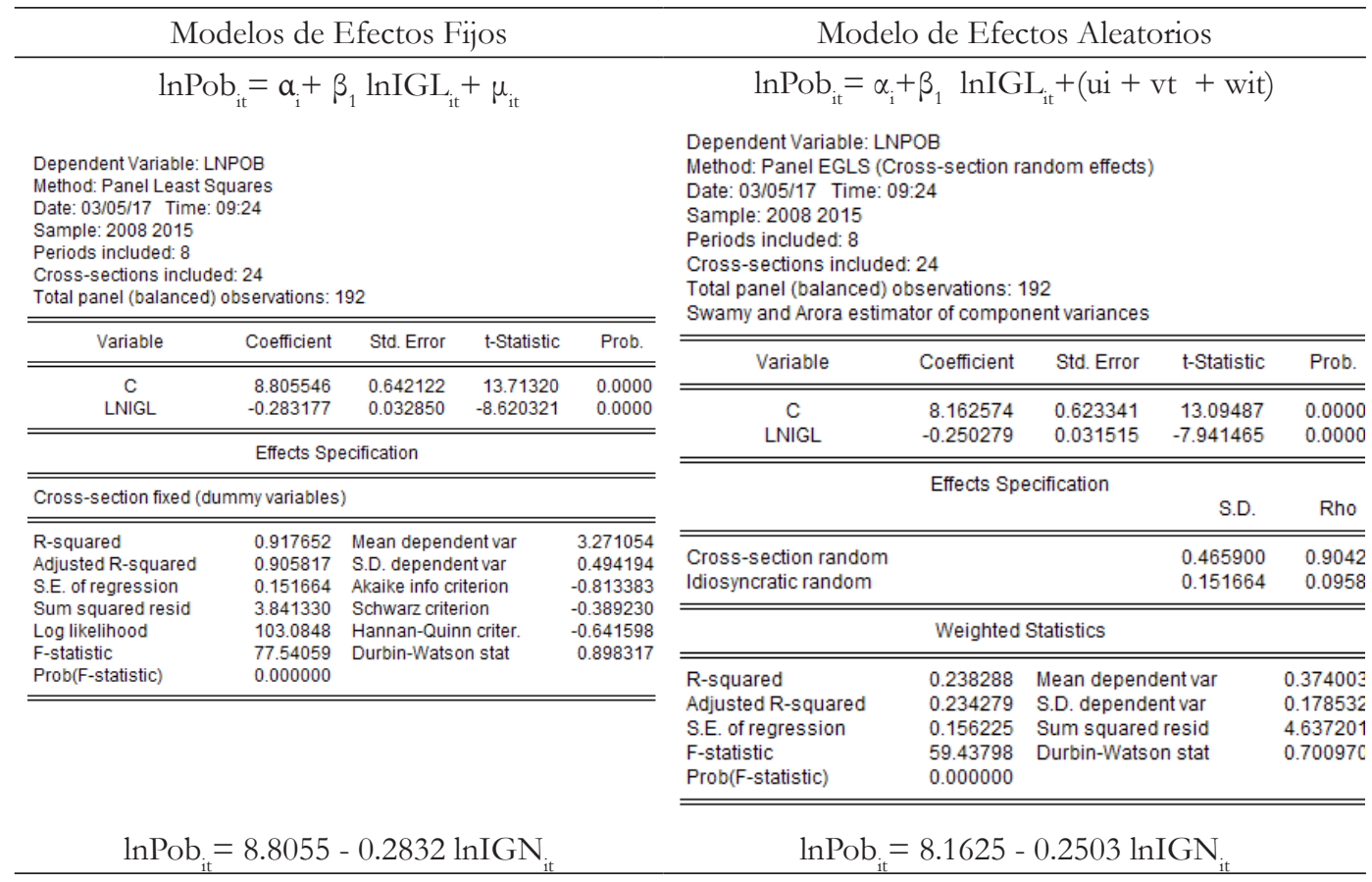

Test de Hausman

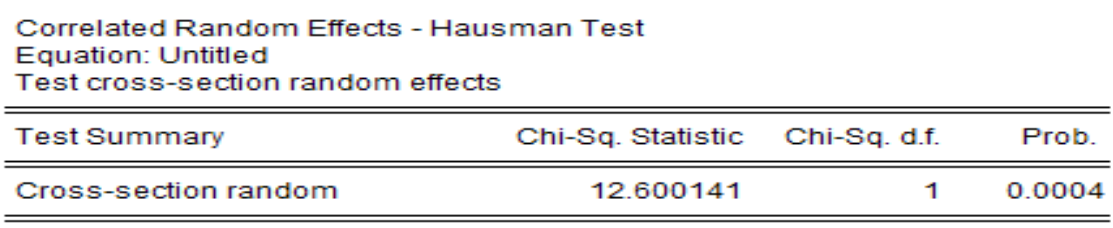

Cross-section random effects test comparisons:

\begin{tabular}{ccccc} 
Variable & Fixed & Random & Var(Diff.) & Prob. \\
\hline \hline LNIGL & -0.283177 & -0.250279 & 0.000086 & 0.0004 \\
\hline \hline
\end{tabular}

De acuerdo a los resultados del Test de Hausman, el modelo aceptado es el modelo de Efectos Fijos. Prob $=0,0004<0,05$

\section{DISCUSIÓN}

El Estado, tal como señala Hernández (2010), es un agente redistribuidor de la riqueza y se orienta básicamente a proveer los recursos necesarios para que estas sean empleadas en los diferentes niveles de gobierno y que sirvan para implementar diferentes acciones tendientes a reducir la pobreza.

La inversión del gobierno nacional, aquella que, desde la sede central, el gobierno realiza en los departamentos contribuye a la reducción de la pobreza, a través del aumento de los ingresos de la población, lo que a su vez permite un mayor gasto de consumo y con ello un mejoramiento de la calidad de vida. Como la pobreza es medida a través 
de las NBI, entonces podemos señalar y de acuerdo a los resultados del modelo que la inversión realizada ha contribuido a la reducción de la pobreza estructural.

Los diferentes niveles de gobierno que interactúan en la economía, tanto nacional como regional y local, inciden de manera diferenciada en su contribución a la reducción de la pobreza; se observa que la inversión de los gobiernos locales tiene mayor efectividad en la inversión porque los resultados señalan una mayor contribución a ello. Los gobiernos locales por estar en mayor cercanía y contacto con la población generan mayor cantidad de empleo, estos a su vez un mayor nivel de ingreso y con ello se mejora la calidad de vida de la población.

Cuando interactúan la inversión nacional, regional y local observamos que la IGR tiene signo positivo lo que significa que hay una superposición de la inversión en sus diferentes niveles. La falta de políticas públicas de inversión, la falta de coordinación hace que esta superposición de la inversión no contribuya de manera significativa y coordinada a la reducción de la pobreza en el ámbito departamental. La posición relativa de contribución de la IGR se ve retraída por esta falta de coordinación. Ello se ve reflejado en el modelo solamente con la IGR regional que si refleja una relación inversa, es decir a mayor IGR menor pobreza. Por cada $1 \%$ de IGR la reducción de la pobreza es del $0,0622 \%$.

\section{CONCLUSIONES}

Los resultados de las estimadores del modelo, nos indican que hay una relación inversa entre la inversión del gobierno nacional y de los gobiernos locales con la reducción de la pobreza (el signo negativo de ellos así lo señala), sin embargo, el signo positivo del estimador que acompaña a la IGR, aparentemente señala que esta relación es directa, lo cual contradice la teoría; sin embargo, podemos intentar una explicación en el sentido de que ello se da por una superposición de la IGR y la IGL. No hay políticas definidas ni coordinación entre dichos niveles de gobierno.

La inversión que realiza el gobierno nacional a nivel departamental mediante sus diferentes organismos, contribuyen de manera positiva al reducción de la pobreza, el signo del estimador (negativo) lo confirma. Hay una relación inversa entre la inversión del Gobierno Nacional y la pobreza. Los incrementos de la inversión del Gobierno Nacional a nivel departamental en un $1 \%$, contribuye a la reducción de la pobreza en un $0,02773 \%$.

La inversión del gobierno regional cuando es relacionada solo con la pobreza, confirma lo señalado por la teoría, hay una relación inversa entre ellas, así por cada $1 \%$ de aumento en la IGR, la pobreza se reduce en $0,0622 \%$. 
La inversión de los gobierno locales tiene una mayor incidencia en la reducción de la pobreza, su cercanía a la problemática local contribuye a ello, así por cada $1 \%$ de aumento en la inversión del Gobierno Local la pobreza se reduce en un 0,2832 \%.

\section{AGRADECIMIENTOS}

A la Universidad Nacional Santiago Antúnez de Mayolo, por las facilidades financieras en la realización del presente trabajo de investigación. Los errores son de exclusiva responsabilidad del equipo investigador.

\section{REFERENCIAS BIBLIOGRÁFICAS}

Adrianzén, Carlos. 2013. «Crecimiento y pobreza en el Perú: 2001-2011». Revista de Economía y Derecho, Vol. 10, Nº 37.

Agénor, Piero y Moreno-Dodson, Blanca. 2006. "Public Infrastructure and Growth: New Channels and Policy Implications”. Policy Research Working Paper Series 4064, World Bank.

Banco Central de Reserva del Perú. Información Regional. Perú: <http://www.bcrp.gob. pe/estadisticas/informacion-regional.html> [Consulta: 20-12-2016].

Durán, Eduardo y Saavedra, Eduardo. 2014. Calidad de la infraestructura y pobreza en América Latina. ILADES.

Feres, Juan y Xavier, Mancero. 2001. "Enfoques para la medición de la pobreza. Breve revisión de la literatura”. CEPAL.

Ford, Ricardo y Paredes, Hector. 2015. Inversión pública y descentralización: sus efectos sobre la pobreza rural en la última década. GRADE. Documento de Investigación 76. Lima, Perú.

Hernández, José. 2010. Inversión pública y crecimiento económico: Hacia una nueva perspectiva de la función del gobierno. Economía: Teoría y práctica, núm. 33, pp. 59-95. México D.F.

Instituto Nacional de Estadística e Informática. Estadísticas Sociales. Perú: <https:// www.inei.gob.pe/estadisticas/indice-tematico/sociales $>$ [Consulta 20-12-2016].

Instituto Peruano de Economía. 2006. Inversión privada y pública en infraestructura en el Perú: el camino para reducir la pobreza. Por encargo de ADEPSEP y CAPECO.

Oates, Wallace E. 1972. Fiscal federalism. Nueva York: Harcourt Brace. 
| Jorge Manrique y Jéssica Polonio

Universidad del Pacífico (UP). 2010. Balance de la Inversión Pública: avances y desafíos para consolidar la competitividad y el bienestar de la población. Por encargo del MEF.

Recibido: $10 / 07 / 17$

Aceptado: 25/10/17

\section{Correspondencia}

Jorge Manrique Cáceres

j.manrique.c@hotmail.com 